\title{
3D hydrodynamic simulations of colliding winds in V444 Cygni
}

\author{
Julian M. Pittard and Ian R. Stevens \\ The University of Birmingham, Edgbaston, Birmingham B15 2TT, UK
}

\begin{abstract}
We report on recent developments in simulating colliding wind Xray emission, and present a 3D model of the WR binary V444 Cyg (WN5+O6) which self-consistently includes radiative driving.
\end{abstract}

\section{Introduction}

The complex nature of the X-ray emission from colliding stellar winds requires sophisticated models which can directly address the multi-temperature, multiabsorption-level nature of the collision shock. Normal single- or two-temperature Raymond-Smith fits are not up to this task, and for a proper investigation of these objects hydrodynamical models must be used. The first interpretation of the X-ray emission from colliding stellar winds involving the use of such models was presented by Luo, McCray \& Mac Low (1990). Major improvements in this method were made by Stevens, Blondin \& Pollock (1992), and in the last five years the hydrodynamical models have become increasingly sophisticated and realistic. The most important developments have concerned studies of the interaction of the radiation fields on the stellar winds of close binary systems (Stevens \& Pollock 1994; Gayley, Owocki \& Cranmer 1997), resulting in the first realistic simulation of the wind collision in an eccentric binary (Pittard 1998).

As the quality of high-spectral-resolution X-ray data improves, RaymondSmith models will become further redundant and more realistic models will become increasingly necessary. Further observations and development are expected to improve the accuracy of mass-loss rate determinations through X-ray spectroscopy (Stevens et al. 1996), a method which should revolutionize the analysis of X-ray spectra from colliding wind systems. Conventional RaymondSmith fits will never be the same again.

\section{Recent work and conclusions}

To further this end, we have recently completed the development of a threedimensional hydrodynamic code which self-consistently includes radiative driving. Preliminary investigations are now underway, and in Fig. 1 we show some density plots of a simulation of V444 Cygni.

We find that the dynamics of the wind collision are altered depending on how we code the radiative-driving force. We use the normal Castor, Abbott \& Klein $(1975$, hereafter CAK) power-law approximation, but with two stars it is not clear which set of parameters to use when we consider the interaction of one 

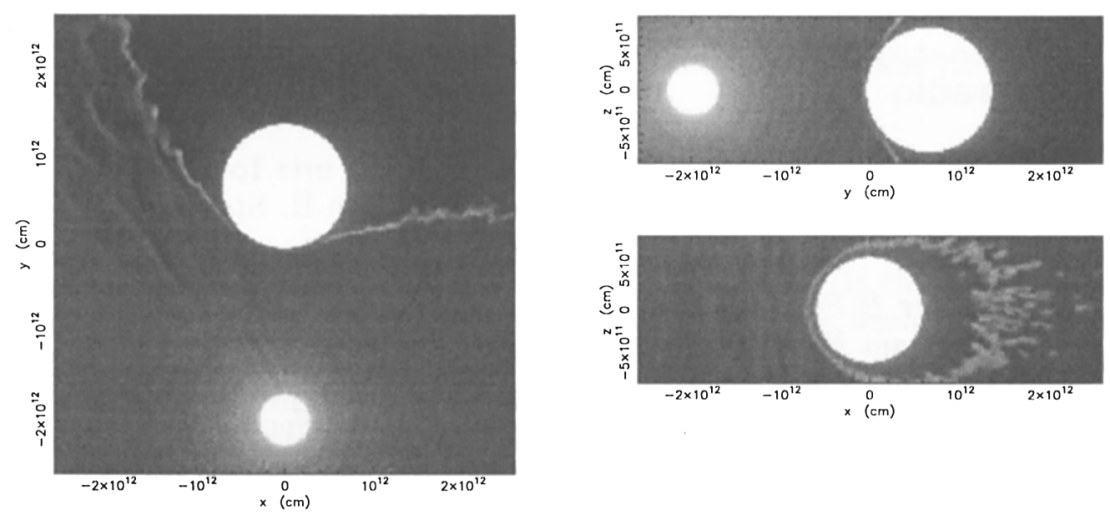

Figure 1. 3D hydrodynamical simulation of the colliding winds in V444 Cygni. All plots shown are gray-scale density. Left: the orbital plane. The WR star is at the bottom, the O-type companion is at the top, and the orbital motion is counter-clockwise. The imbalance in the wind momentum ratios causes the colliding wind shock to collapse onto the surface of the $O$ star. The skew of the shock due to the orbital motion is also clearly visible. Upper right: the yz-plane through the centers of the stars. Lower right: A plane almost tangential to the surface of the shock. This plot clearly shows the 'stipply' nature of the shock surface.

star's radiation field with the other star's wind. In our simulation of V444 Cyg, sudden radiative braking is much more effective if the CAK parameters are fixed to the wind. The difference in the amount of braking has substantial implications for the X-ray emission from the wind collision.

Preliminary results show that the orbital motion can also have a large effect on the observed X-ray spectra. We find that at certain phases, the observed Xray emission at energies $\mathrm{E} \leq 1.0 \mathrm{keV}$ is strongly dependant on the position of the shock in the simulation, which is skewed if orbital motion is incorporated into the models. Hence, to realistically model the X-ray emission from close binary systems we should include this effect.

\section{References}

Castor, J.L., Abbott, D.C., Klein, R.I. 1975, ApJ 195, 157

Gayley, K.G., Owocki, S.P., Cranmer, S.R. 1997, ApJ 475, 786

Luo, D., McCray, R., Mac Low, M. 1990, ApJ 362, 267

Pittard, J.M. 1998, MNRAS 300, 479

Stevens, I.R., Blondin, J.M., Pollock, A.M.T. 1992, ApJ 386, 265

Stevens, I.R., Pollock, A.M.T. 1994, MNRAS 269, 226

Stevens, I.R., et al. 1996, MNRAS 283, 569 